\title{
Towards Practical Channel Reciprocity Exploitation: Relative Calibration in the Presence of Frequency Offset
}

\author{
Maxime Guillaud \\ Vienna University of Technology \\ Institute of Telecommunications \\ Vienna, Austria \\ email: guillaudetuwien.ac.at
}

\author{
Florian Kaltenberger \\ Eurecom \\ Mobile Communications Department \\ Sophia-Antipolis, France \\ email: Florian.Kaltenberger@eurecom.fr
}

\begin{abstract}
Relative calibration has been proposed as a simple way to practically exploit the reciprocity of the wireless channel. It is based on a simple convolutional model for the relationship between the channel impulse responses in both directions, and accounts for the discrepancies between transmit and receive radiofrequency components, without the need for specific calibration hardware. However, the relative calibration methods developed so far have been shown to lack robustness with respect to frequency offset between the devices on both sides of the considered channel. In this article, we introduce a relative calibration algorithm that properly deals with the presence of frequency offset. We verify its robustness and assess its performance through simulations, and validate experimentally the proposed model on measured channels.
\end{abstract}

\section{INTRODUCTION}

The symmetry of the electromagnetic propagation channel w.r.t. exchange of the roles of the transmitter and receiver, or reciprocity, is often cited in the literature as a convenient way to obtain channel state information at the transmitter (CSIT) without requiring a feedback link. Indeed, in systems where the channel is used in both directions using a time-division duplexing scheme, the channel estimates obtained from the received signals can theoretically be used to infer the state of the channel during a subsequent transmission, provided that the channel does not change too fast.

However, this symmetry is in practice disturbed by the characteristics of the radio-frequency (RF) circuitry of the transmitter and receiver. Indeed, the channel representation which is used typically by digital signal processors in communication applications is a combination of the characteristics of the digital-to-analog converters and power amplifiers at the transmit side, the antennas on both transmit and receive sides, the electromagnetic channel itself, and the characteristics of the low-noise amplifier and analog-to-digital converters at the receive side. Although the electromagnetic channel between the antennas is demonstrably reciprocal, (see for instance [1] and references therein), the RF circuits on both sides are usually not identical. This indicates that exchanging the roles of the transmitter and the receiver would actually affect the channel measured by the digital signal processing algorithms.
Various solutions to this issue have been proposed. One of them is the calibration of each RF circuit involved [2], requiring additional hardware. Another alternative, limited to lowpower transmission, is to use a specially crafted transceiver where the same op-amp is used for both transmitting and receiving [3]. A third alternative [4], [5], termed relative calibration, achieves the same effect as normal calibration without the requirement for extra hardware. Assuming that the impairments due to imperfect RF chains can be modelled as linear time-invariant (LTI) filters, it was shown in [4] that the channels measured in both directions could be related by a simple convolution operation, thus alleviating the need for hardware-based calibration.

Several recent experimental works, such as [6], [7], have successfully demonstrated the use of relative calibration. They have in common that calibration is formulated using a frequency-flat channel representation. In the presence of a frequency selective channel, calibration must be performed independently for each subcarrier, resulting in an over-parameterization and possibly reducing the calibration accuracy. The deconvolution-based calibration algorithm from [4], conversely, is formulated in the time domain, and does not suffer from this over-parameterization. It was observed by the authors however that it performs poorly in the presence of frequency offset between the two sides of the link. The present paper introduces an improved deconvolution method that achieves accurate relative calibration in the presence of frequency offset. The method is validated both with synthetic data and with experimental data collected the with OpenAirInterface LTE testbed.

This article is organized as follows: the system model and the principle of relative calibration are presented in Sections [II and III In Section IV] we introduce a calibration algorithm robust to frequency offset. It is validated on synthetic data in Section V, and on experimental data in Section VI.

Notations: We let $*$ denotes the convolution operator, $\operatorname{Re}\{\cdot\}$ and $\operatorname{Im}\{\cdot\}$ denote respectively the real and imaginary parts of the argument. $\|\cdot\|_{\mathrm{F}}$ is the Frobenius norm, and ${ }^{H}$ denotes the Hermitian transpose operation. I is the identity matrix. 


\section{SySTEM MODEL}

We briefly recall the reciprocity model of [4], which relies on modelling the measured channel as the cascade of filters modeling respectively the transmit circuitry, electromagnetic propagation path, and receive circuitry. Assuming bidirectional communications between two devices denoted by $\mathrm{A}$ and $\mathrm{B}$, the bi-directional channel is modeled as two fading additive white Gaussian noise (AWGN) channels, see Fig. 1.

According to this model, the channels perceived by transceivers in the digital domain (e.g. through classical channel estimation techniques), is comprised of the effective electromagnetic channel $(C(t))$, assumed identical in both directions, and filters modeling the imperfections of the power amplifiers $\left(T_{A}, T_{B}\right)$ and low-noise amplifiers $\left(R_{A}, R_{B}\right)$.

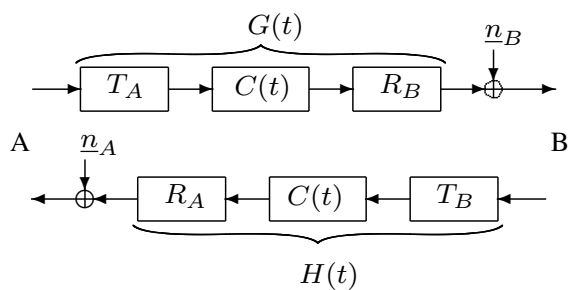

Fig. 1. Reciprocity model

From A to B, the channel impulse response is modeled as $G(t, \tau)=R_{B}(\tau) * C(t, \tau) * T_{A}(\tau)$, where the convolutions are over the delay domain, i.e. variable $\tau$. In the opposite direction, from $\mathrm{B}$ to $\mathrm{A}$, the impulse response is $H(t, \tau)=$ $R_{A}(\tau) * C(t, \tau) * T_{B}(\tau)$. Note that both $G$ and $H$ are assumed to be functions of the same $C$. Note also that $G$ and $H$ are functions (through $C(t)$ ) of the channel estimation epoch $t$, while $T_{A}, T_{B}, R_{A}$ and $R_{B}$ are assumed to remain constant over the duration of the experiment.

Note that this model is valid both for single-antenna and for multiple-antenna systems (in the latter case, the considered filters have vector inputs and/or outputs). In the next section, we focus exclusively on the case of single-antenna nodes, i.e. all channels are be assumed to be Single-Input SingleOutput (SISO). Note however that any $M \times N$ MultipleInput Multiple-Output (MIMO) channel can be regarded as a collection of $M N$ SISO channels for the purpose of relative calibration. Therefore, the method proposed here is also applicable to MIMO systems, provided that relative calibration is performed independently for each transmit/receive antenna pair, as demonstrated in Section $\mathrm{V}$

\section{RELATIVE CALIBRATION}

Under the SISO channel assumption, convolution of linear filters is commutative. As a consequence, $G$ and $H$ can be related to each other directly through

$$
G(t, \tau)=H(t, \tau) * R(\tau),
$$

where $R(\tau) \triangleq R_{B}(\tau) * R_{A}(\tau)^{-1} * T_{B}(\tau)^{-1} * T_{A}(\tau)$. Note that the existence of the inverse filters is generally not an issue here, since the involved filters should be exempt of zeros by design.
Relative calibration consists in estimating $R(\tau)$ (through deconvolution) from one or several successive bi-directiona ${ }^{1}$ channel measurements $\left(G\left(t_{i}\right), H\left(t_{i}\right)\right)_{i=1 \ldots K}$, where $K$ denotes the number of measurements used for the training phase. Once this estimate $\hat{R}(\tau)$ is available, it can be used at a later time $t^{\prime}$ to compute a reciprocity-based estimate of $G\left(t^{\prime}, \tau\right)$ based on $H\left(t^{\prime}, \tau\right)$ :

$$
\tilde{G}\left(t^{\prime}, \tau\right)=H\left(t^{\prime}, \tau\right) * \hat{R}(\tau) .
$$

\section{A. TLS Deconvolution method}

Let $\mathbf{g}, \mathbf{h}$ and $\mathbf{r}$ denote vectors containing the sampled versions of impulse responses $G(\tau), H(\tau)$ and $R(\tau)$ respectively, and note that the convolution (11) can equivalently be written as a vector-matrix product

$$
\underbrace{\left[\begin{array}{c}
g_{1} \\
g_{2} \\
\vdots
\end{array}\right]}_{\mathbf{g}}=\underbrace{\left[\begin{array}{cccc}
h_{1} & & & \\
h_{2} & h_{1} & & \\
h_{3} & h_{2} & h_{1} & \\
\vdots & \vdots & & \ddots
\end{array}\right]}_{\mathbf{H}} \cdot \underbrace{\left[\begin{array}{c}
r_{1} \\
r_{2} \\
\vdots
\end{array}\right]}_{\mathbf{r}},
$$

where $\mathbf{H}$ is a Toeplitz matrix defined by its first column $\mathbf{h}$.

Total least-squares (TLS) deconvolution enables to estimate $\mathbf{r}$ while accounting for imperfect channel estimates. Denoting $\mathbf{g}=\widehat{\mathbf{g}}+\mathbf{e}, \mathbf{h}=\widehat{\mathbf{h}}+\mathbf{f}$, where $\mathbf{e}$ and $\mathbf{f}$ represent the error terms, and $\widehat{\mathrm{g}}$ and $\widehat{\mathbf{h}}$ are the estimated channels, notice that (3) is equivalent to

$$
\widehat{\mathbf{g}}+\mathbf{e}=(\widehat{\mathbf{H}}+\mathbf{F}) \cdot \mathbf{r}
$$

where $\mathbf{F}$ is Toeplitz with first column $\mathbf{f}$. TLS estimation of $R$ consists in solving

$$
\widehat{\mathbf{r}}=\arg \min _{\mathbf{e}, \mathbf{f}, \mathbf{r}: \mathbf{e}=(\widehat{\mathbf{H}}+\mathbf{F}) \mathbf{r}-\widehat{\mathbf{g}}}\|\mathbf{e}\|_{\mathrm{F}}^{2}+\|\mathbf{f}\|_{\mathrm{F}}^{2} .
$$

Intuitively, it consists in finding the correction terms $\mathbf{e}$ and $\mathbf{f}$ with minimal Euclidean norm that allow (4) to be fulfilled for some $r$. An iterative solution to the above deconvolution problem was proposed in [8].

\section{Multi-MeAsurement RECIPROCITy ESTIMATION}

If multiple channel impulse responses pairs $\left(\mathbf{g}^{(k)}, \mathbf{h}^{(k)}\right)$, $k=0 \ldots K$ (obtained at time instants $t_{0}<\ldots<t_{K}$ ) are available for the training phase, it is expected that they can be used jointly to provide a better estimate of $R(\tau)$. In particular, it is clear from (1) that $R(\tau)$ can not be estimated reliably for frequencies where $H$ (and consequently $G$ ) have zeros. If multiple measurements (assumed to be taken from independent fades of the channel) can be used, the likelihood that all channels exhibit a zero at the same frequency decreases.

\footnotetext{
${ }^{1}$ Although TDD operation does not, strictly speaking, enable simultaneous bi-directional channel sounding, it is sufficient for our purpose that the delay between the two measurements remains well below the channel coherence time, which is a practical assumption.
} 


\section{A. Multiple channel measurements model}

An attempt at joint deconvolution of multiple bi-directional channel measurements was proposed in [4]. Experimental investigations by the authors, however, have shown that the algorithm in [4] exhibits poor performance due to its inadequate handling of frequency offset. Therefore, we introduce the following model, where arbitrary rotations between channel measurements model the effects of frequency offset:

$$
\left[\begin{array}{c}
\mathbf{g}^{(0)} \\
e^{\jmath \phi_{1}} \mathbf{g}^{(1)} \\
\vdots \\
e^{\jmath \phi_{K}} \mathbf{g}^{(K)}
\end{array}\right]=\left[\begin{array}{c}
\mathbf{H}^{(0)} \\
\mathbf{H}^{(1)} \\
\vdots \\
\mathbf{H}^{(K)}
\end{array}\right] \cdot \mathbf{r},
$$

where $\phi_{1} \ldots \phi_{K}$ are real angles, and the $e^{\jmath \phi_{k}}$ represent the rotation accumulated between measurements 0 and $k$ due to the clock offsets. $\mathbf{H}^{(k)}$ denotes the Toeplitz matrix with first column $\mathbf{h}^{(k)}$, similar to 3 .

A few remarks about the above model are in order. Letting $f_{o}$ denote the frequency offset between transmitter and receiver, one has that $\phi_{k}=2 \pi f_{o}\left(t_{k}-t_{0}\right), k=1 \ldots K$, i.e. we could use a unique unknown variable $f_{o}$ instead of $\phi_{1} \ldots \phi_{K}$ in (6). However, note that

- the $\phi_{k}$ in (6) are only significant modulo $2 \pi$,

- if $t_{k}-t_{0}$ gets large, the estimation accuracy of $f_{o}$ must be extremely high in order to guarantee acceptable accuracy on the $\phi_{k}$. Indeed, let $\widehat{f}_{o}=f_{o}+\epsilon$ and $\widehat{\phi}_{k}=2 \pi \widehat{f}_{o}\left(t_{k}-t_{0}\right)$, thus $\widehat{\phi}_{k}-\phi_{k}=2 \pi \epsilon\left(t_{k}-t_{0}\right)$. Since the $\phi_{k}$ are only significant modulo $2 \pi$, it is clear that the desired accuracy lies in the regime where $\left|\widehat{\phi}_{k}-\phi_{k}\right| \ll 2 \pi$, i.e. $\epsilon \ll \frac{1}{t_{k}-t_{0}}$. In practice, since $t_{k}-t_{k-1}$ should be larger than the channel coherence time for the reasons mentioned above, we expect $t_{K}-t_{0}$ to be measured in seconds. It is therefore clear that the sub- $\mathrm{Hz}$ accuracy required for $\widehat{f}_{o}$ is out of reach for state-of-the-art frequency offset estimators.

In light of the above, we choose to consider the overparameterized model in (6), and treat $\phi_{1} \ldots \phi_{K}$ as nuisance parameters. We now introduce a noisy version of (6), formulate relative calibration as a structured TLS estimation problem, and propose an iterative solution.

\section{B. Noisy measurements and structured TLS formulation}

Let us introduce the channel estimation errors by decomposing $\mathbf{g}^{(k)}$ and $\mathbf{h}^{(k)}$ into $\mathbf{g}^{(k)}=\widehat{\mathbf{g}}_{k}+\mathbf{e}_{k}$ and $\mathbf{H}^{(k)}=\widehat{\mathbf{H}}_{k}+\mathbf{F}_{k}$, where $\widehat{\mathbf{g}}_{k}$ and $\widehat{\mathbf{H}}_{k}$ denote the measured channels, while $\mathbf{e}_{k}$ and the Toeplitz matrix $\mathbf{F}_{k}$ (with first column $\mathbf{f}_{k}$ ) denote the estimation error terms. Since the true impulse responses $\mathbf{g}^{(k)}$ and $\mathbf{H}^{(k)}$ are assumed to fulfill $(6)$, we have

$$
e^{\jmath \phi_{k}}\left(\widehat{\mathbf{g}}_{k}+\mathbf{e}_{k}\right)=\left(\widehat{\mathbf{H}}_{k}+\mathbf{F}_{k}\right) \mathbf{r} \quad \forall k=0 \ldots K,
$$

where we take the reference phase $\phi_{0}=0$ by convention.
Again, TLS estimation consists in minimizing the Euclidean norm of the correction terms $\mathbf{e}_{k}$ and $\mathbf{f}_{k}$ required to make (7) true for some $\mathbf{r}$ and $\phi_{1}, \ldots, \phi_{k}$, i.e.

$$
\begin{aligned}
\widehat{\mathbf{r}}=\arg \min _{\underline{\mathbf{e}, \underline{\mathbf{f}}, \mathbf{r}, \Phi}} & \sum_{k=0}^{K}\left\|\mathbf{e}_{k}\right\|_{\mathrm{F}}^{2}+\left\|\mathbf{f}_{k}\right\|_{\mathrm{F}}^{2} \\
\text { subject to } & \mathbf{e}_{k}=e^{-\jmath \phi_{k}}\left(\widehat{\mathbf{H}}_{k}+\mathbf{F}_{k}\right) \mathbf{r}-\widehat{\mathbf{g}}_{k} \quad \forall k,
\end{aligned}
$$

where $\underline{\mathbf{e}}=\left[\mathbf{e}_{0}^{T} \ldots \mathbf{e}_{K}^{T}\right]^{T}, \underline{\mathbf{f}}=\left[\mathbf{f}_{0}^{T} \ldots \mathbf{f}_{K}^{T}\right]^{T}$, and $\Phi=$ $\left(\phi_{1}, \ldots, \phi_{k}\right)$.

\section{Proposed iterative solution}

For a given set of channel measurements, i.e. for given $\widehat{\mathbf{g}}_{k}$ and $\widehat{\mathbf{H}}_{k}, \mathbf{e}_{k}$ can be written as a function of the other the optimization parameters, i.e.

$$
\mathbf{e}_{k}\left(\mathbf{f}_{k}, \mathbf{r}, \phi_{k}\right)=e^{-\jmath \phi_{k}}\left(\widehat{\mathbf{H}}_{k}+\mathbf{F}_{k}\right) \mathbf{r}-\widehat{\mathbf{g}}_{k} .
$$

The non-linear aspect of this function makes the problem (8) difficult to tackle. Following [8], we linearize $\mathbf{e}_{k}$ for some small variations $\Delta \mathbf{f}_{k}, \Delta \mathbf{r}$ and $\Delta \phi_{k}$ of the parameters:

$$
\begin{aligned}
& \mathbf{e}_{k}\left(\mathbf{f}_{k}+\Delta \mathbf{f}_{k}, \mathbf{r}+\Delta \mathbf{r}, \phi_{k}+\Delta \phi_{k}\right) \\
& =\mathbf{e}_{k}\left(\mathbf{f}_{k}, \mathbf{r}, \phi_{k}\right)+\Delta \mathbf{e}_{k}+o\left(\left\|\Delta \phi_{k}\right\|+\left\|\Delta \mathbf{f}_{k}\right\|+\|\Delta \mathbf{r}\|\right),
\end{aligned}
$$

where

$$
\begin{aligned}
\Delta \mathbf{e}_{k}= & e^{-\jmath \phi_{k}}\left(\widehat{\mathbf{H}}_{k}+\mathbf{F}_{k}\right) \Delta \mathbf{r}+e^{-\jmath \phi_{k}} \Delta \mathbf{F}_{k} \mathbf{r} \\
& -\jmath e^{-\jmath \phi_{k}}\left(\widehat{\mathbf{H}}_{k}+\mathbf{F}_{k}\right) \mathbf{r} \Delta \phi_{k} .
\end{aligned}
$$

Note that $\Delta \phi_{0}=0$ by convention since $\phi_{0}$ is fixed, allowing (11) to hold for all $k$ including $k=0$. Let us denote $\Delta \underline{\mathbf{e}}=\left[\Delta \mathbf{e}_{0}^{T} \ldots \Delta \mathbf{e}_{K}^{T}\right]^{T}, \Delta \underline{\mathbf{f}}=\left[\Delta \mathbf{f}_{0}^{T} \ldots \Delta \mathbf{f}_{K}^{T}\right]^{T}$ and $\Delta \Phi=\left(\Delta \phi_{1}, \ldots, \Delta \phi_{k}\right)$.

We propose to solve iteratively the linearized version of the optimization problem (8) around the current values of $\underline{\mathbf{e}}, \underline{\mathbf{f}}, \mathbf{r}$ and $\Phi$, i.e. at each iteration we solve

$$
\begin{aligned}
\min _{\Delta \underline{\mathbf{e}}, \Delta \underline{\mathbf{f}}, \Delta \mathbf{r}, \Delta \Phi} & \sum_{k=0}^{K}\left\|\mathbf{e}_{k}+\Delta \mathbf{e}_{k}\right\|_{\mathrm{F}}^{2}+\left\|\mathbf{f}_{k}+\Delta \mathbf{f}_{k}\right\|_{\mathrm{F}}^{2} \\
\text { subject to } & 111 \quad \forall k=0, \ldots, K .
\end{aligned}
$$

We now describe how (12) can be solved efficiently. Clearly, $\mathbf{e}_{k}+\Delta \mathbf{e}_{k}$ and $e^{\jmath \phi_{k}}\left(\mathbf{e}_{k}+\Delta \mathbf{e}_{k}\right)$ have the same Frobenius norm, therefore the objective function of $(12)$ can be rewritten as

$$
\sum_{k=0}^{K}\left\|\mathbf{e}_{k}+\Delta \mathbf{e}_{k}\right\|_{\mathrm{F}}^{2}+\left\|\mathbf{f}_{k}+\Delta \mathbf{f}_{k}\right\|_{\mathrm{F}}^{2}=\left\|\left[\begin{array}{c}
K(\Phi)(\underline{\mathbf{e}}+\Delta \underline{\mathbf{e}}) \\
\underline{\mathbf{f}}+\Delta \underline{\mathbf{f}}
\end{array}\right]\right\|_{\mathrm{F}}^{2},
$$

where $K(\Phi)=\operatorname{diag}\left(\mathrm{I}, e^{\jmath \phi_{1}} \mathrm{I}, \ldots, e^{\jmath \phi_{K}} \mathrm{I}\right)$.

Let $\mathbf{R}$ be the Toeplitz matrix with first column $\mathbf{r}$, i.e. it is such that $\Delta \mathbf{F}_{k} \mathbf{r}=\mathbf{R} \Delta \mathbf{f}_{k}$. From (11), we have that $e^{\jmath \phi_{k}} \Delta \mathbf{e}_{k}=$ $\left(\widehat{\mathbf{H}}_{k}+\mathbf{F}_{k}\right) \Delta \mathbf{r}+\mathbf{R} \Delta \mathbf{f}_{k}-\jmath\left(\widehat{\mathbf{H}}_{k}+\mathbf{F}_{k}\right) \mathbf{r} \Delta \phi_{k}$, and therefore 


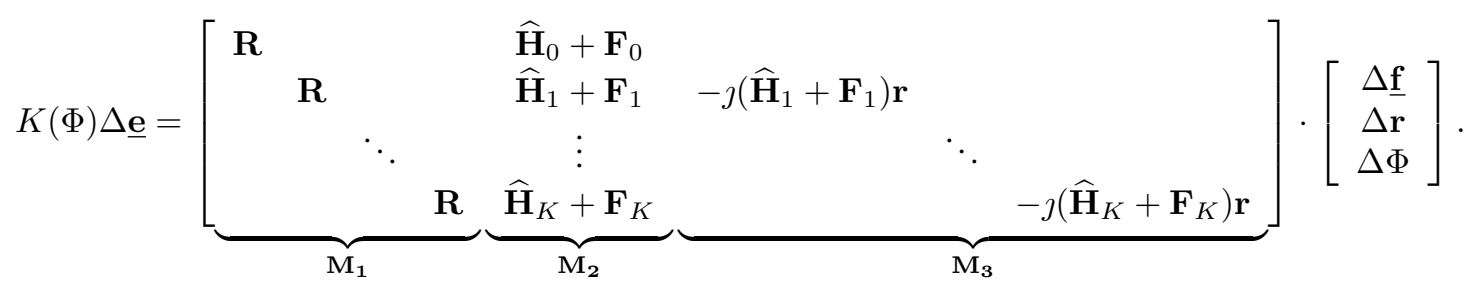

the product $K(\Phi) \Delta \underline{\mathbf{e}}$ can be written as in (14) (above). Thus,

$$
\left[\begin{array}{c}
K(\Phi)(\underline{\mathbf{e}}+\Delta \underline{\mathbf{e}}) \\
\underline{\mathbf{f}}+\Delta \underline{\mathbf{f}}
\end{array}\right]=\left[\begin{array}{c}
K(\Phi) \underline{\mathbf{e}} \\
\underline{\mathbf{f}}
\end{array}\right]+\left[\begin{array}{ccc}
\mathrm{M}_{1} & \mathrm{M}_{2} & \mathrm{M}_{3} \\
\mathrm{I} & \mathbf{0} & \mathbf{0}
\end{array}\right] \cdot\left[\begin{array}{c}
\Delta \underline{\mathbf{f}} \\
\Delta \mathbf{r} \\
\Delta \Phi
\end{array}\right],
$$

where $\mathrm{M}_{1}, \mathrm{M}_{2}$ and $\mathrm{M}_{3}$ are defined in (14). With these notations, 12 becomes a least-squares problem:

$$
\min _{\Delta \underline{\mathbf{f}}, \Delta \mathbf{r}, \Delta \Phi}\left\|\left[\begin{array}{c}
K(\Phi) \underline{\mathbf{e}} \\
\underline{\mathbf{f}}
\end{array}\right]+\left[\begin{array}{ccc}
\mathrm{M}_{1} & \mathrm{M}_{2} & \mathrm{M}_{3} \\
\mathrm{I} & \mathbf{0} & \mathbf{0}
\end{array}\right] \cdot\left[\begin{array}{c}
\Delta \underline{\mathbf{f}} \\
\Delta \underline{\mathbf{r}} \\
\Delta \Phi
\end{array}\right]\right\|_{(16)}^{2} .
$$

Note however that $\Delta \Phi$ is real, while $\Delta \mathbf{f}$ and $\Delta \mathbf{r}$ are in general complex. The constraint $\operatorname{Im}\{\Delta \Phi\}=0$ makes the above least-squares problem non linear in the complex field. This issue is lifted by separating the real and imaginary parts of the complex variables, yielding the real least-squares problem (17) (next page).

The proposed algorithm consists in iteratively solving (17):

$$
\begin{aligned}
& \text { initialization: } \underline{\mathbf{f}}=\underline{0}, \underline{\mathbf{r}}=\underline{1}, \underline{\boldsymbol{\Phi}}=\underline{0} \\
& \text { repeat }
\end{aligned}
$$

compute e according to 9

solve the least-squares problem (17)

update $\underline{\mathbf{f}} \leftarrow \underline{\mathbf{f}}+\Delta \underline{\mathbf{f}}, \underline{\mathbf{r}} \leftarrow \underline{\mathbf{r}}+\Delta \underline{\mathbf{r}}, \underline{\mathbf{\Phi}} \leftarrow \underline{\mathbf{\Phi}}+\Delta \underline{\boldsymbol{\Phi}}$

until $\Delta \underline{\mathbf{f}}, \Delta \underline{\mathbf{r}}$ and $\Delta \underline{\boldsymbol{\Phi}}$ are small enough.

Similarly to the original Structured TLS algorithm [8], no proof of convergence nor optimality of the above algorithm could be established. Simulations presented in the next sections however demonstrate adequate performance.

\section{Validation Using Synthetic Data}

In order to validate the algorithm of Section IV-C, a series of simulations was performed on synthetic channel impulse responses generated such that (6) holds exactly. The true reciprocity parameter $\mathbf{r}$ was generated through independent realizations of a random circularly symmetric (c.c.) Gaussian i.i.d. fading process with power delay profile according to Fig. 2(a) For each realization of $\mathbf{r}, K+1=10$ impulse responses $\mathbf{h}^{(k)}$ were drawn independently from a c.c. Gaussian distribution, according to the power delay profile of Fig. 2(b) $\mathbf{g}^{(k)}$ is computed according to (3), and a frequency offset of normalized frequency $2 \cdot 10^{-4}$ is introduced. White c.c. Gaussian noise is added to both impulse responses in order to simulate the effect of estimation error, according to the target channel estimate signal-to-noise ratio (denoted by $\mathrm{SNR}_{\mathrm{C}}$ ). Finally, the resulting noisy impulse responses are

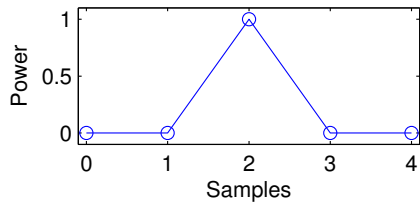

(a) Power delay profile of $\mathbf{r}$

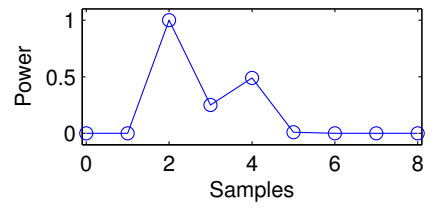

(b) Power delay profile of $\mathbf{h}^{(k)}$
Fig. 2. Power delay profile of the reciprocity parameter and channel impulse responses used for the simulation with synthetic data.

processed by the calibration algorithm to produce $\hat{r}$. This process was repeated for each antenna pair in a 4 transmit, 2-receive antennas MIMO system operating at $10 \mathrm{~dB}$ SNR, after which the obtained relative calibration was utilized to generate CSIT via reciprocity, according to eq. (2). Finally, this CSIT is utilized to perform space-frequency waterfilling over an OFDM transmission with 128 subcarriers, and the achieved mutual information per subcarrier is evaluated. The results are presented in Fig. 3, where we present for comparison the performance obtained with perfect CSIT, with no CSIT (signal i.i.d. in space and frequency), and using the relative calibration relying on per-subcarrier calibration based on a single pair of bi-directional channel measurements [9] (denoted by "simple" reciprocity exploitation).

These results clearly demonstrate that at high $\mathrm{SNR}_{\mathrm{C}}$, relative calibration can provide perfect CSIT, while at the lowest range of $\mathrm{SNR}_{\mathrm{C}}$, relative calibration is unreliable and in fact yields worse performance than no CSIT. Furthermore, for a given $\mathrm{SNR}_{\mathrm{C}}$, the algorithm proposed here provides better CSIT than calibration based on a single channel measurement.

\section{Validation Using Measured Data}

In order to check the validity of the model in (6), and to assess the performance of relative calibration in a practical system, the proposed algorithm was applied to measurements collected using the Eurecom MIMO OpenAir Sounder (EMOS), a subsystem of the OpenAirInterface LTE testbed (http://www.openairinterface.org).

\section{A. EMOS Hardware}

The hardware used for the user equipment (UE) is depicted in Fig. 4 It consists of PC running the software modem, the Express MIMO board, the LIME RF front-end, and an antenna. The base station (eNB) is similar with the difference that the RF front-end and the antennas are duplicated.

Express MIMO is a baseband processing board, which comprises two FPGAs: one Xilinx XC5VLX330 for real- 


$$
\min _{\Delta \underline{\mathbf{f}}, \Delta \mathbf{r}, \Delta \Phi}\left\|\left[\begin{array}{c}
\operatorname{Re}\{K(\Phi) \underline{\mathbf{e}}\} \\
\operatorname{Re}\{\underline{\mathbf{f}}\} \\
\operatorname{Im}\{K(\Phi) \underline{\mathbf{e}}\} \\
\operatorname{Im}\{\underline{\mathbf{f}}\}
\end{array}\right]+\left[\begin{array}{ccccc}
\operatorname{Re}\left\{\mathbf{M}_{\mathbf{1}}\right\} & \operatorname{Re}\left\{\mathbf{M}_{\mathbf{2}}\right\} & -\operatorname{Im}\left\{\mathbf{M}_{\mathbf{1}}\right\} & -\operatorname{Im}\left\{\mathbf{M}_{\mathbf{2}}\right\} & \operatorname{Re}\left\{\mathbf{M}_{\mathbf{3}}\right\} \\
\operatorname{I} & \mathbf{0} & \mathbf{0} & \mathbf{0} & \mathbf{0} \\
\operatorname{Im}\left\{\mathbf{M}_{\mathbf{1}}\right\} & \operatorname{Im}\left\{\mathbf{M}_{\mathbf{2}}\right\} & \operatorname{Re}\left\{\mathbf{M}_{\mathbf{1}}\right\} & \operatorname{Re}\left\{\mathbf{M}_{\mathbf{2}}\right\} & \operatorname{Im}\left\{\mathbf{M}_{\mathbf{3}}\right\} \\
\mathbf{0} & \mathbf{0} & \mathrm{I} & \mathbf{0} & \mathbf{0}
\end{array}\right] \cdot\left[\begin{array}{c}
\operatorname{Re}\{\Delta \mathbf{f}\} \\
\operatorname{Re}\{\Delta \mathbf{r}\} \\
\operatorname{Im}\{\Delta \mathbf{f}\} \\
\operatorname{Im}\{\Delta \mathbf{r}\} \\
\Delta \Phi
\end{array}\right]\right\|_{\mathrm{F}}
$$

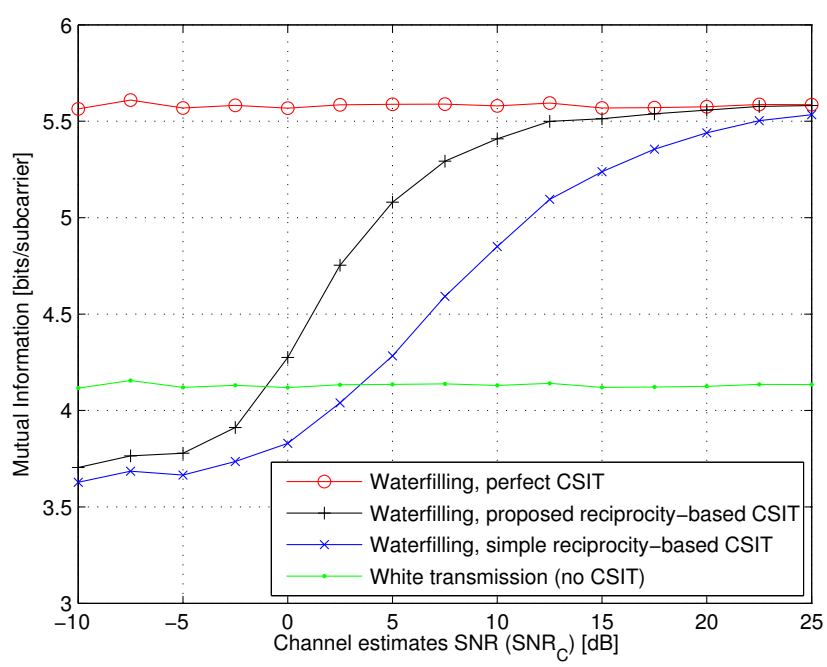

Fig. 3. Mutual information per subcarrier achieved with various sources of CSIT, and without CSIT.

time embedded signal processing applications and one Xilinx XC5VLX110T for control. The card uses an eight-way PCI express interface to communicate with the host PC. The card employs four high-speed A/D and D/A converters from Analog Devices (AD9832) allowing to drive four RF chains using quadrature modulation. In the current setting the ADAC are configured to 7.68 MSPS (corresponding to the LTE $5 \mathrm{MHz}$ bandwidth allocation).

As an RF front-end we have used a custom design based on the LMS6002D evaluation boards from Lime Microsystems. Up to four such front-ends can be connected, each independently tunable from $300 \mathrm{MHz}$ to $3.8 \mathrm{GHz}$ with a maximum output power of $0 \mathrm{dBm}$. However, the current filters limit the

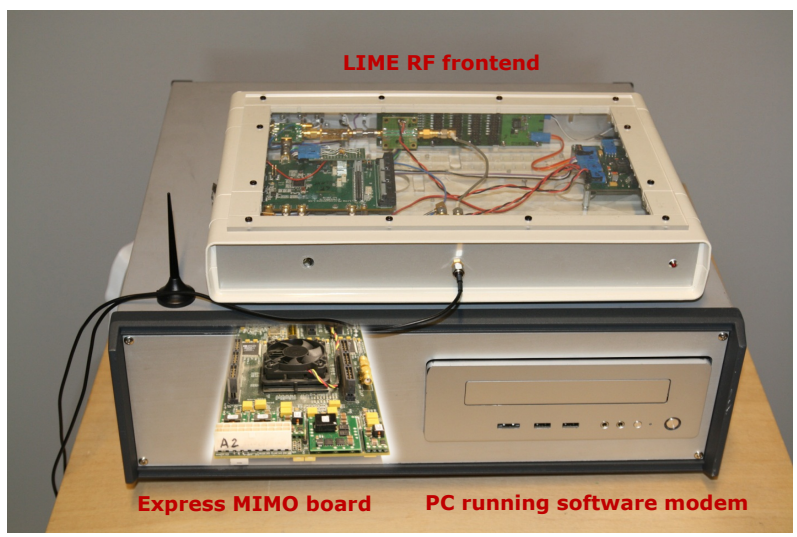

Fig. 4. User equipment (UE) used in the measurements.

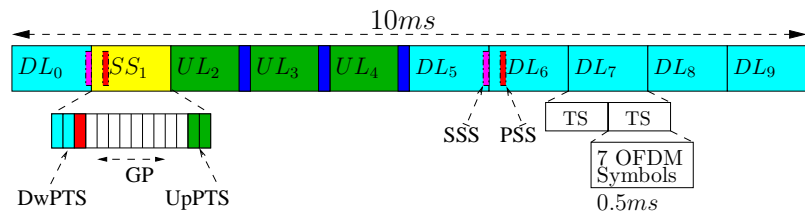

Fig. 5. Frame structure for LTE TDD type 3

carrier frequency to the band around $1.9 \mathrm{GHz}$.

\section{B. Measurement methodology}

a) System overview: A software modem running under the control of the real-time application interface (RTAI) of a standard Linux operating system uses the Express MIMO card for baseband signal acquisition and transmission. The software modem implements a large part of the 3GPP LTE Rel 8.6 standard. For the measurements we used TDD configuration 3, depicted in Fig. 5 It consists of 6 downlink (DL) subframes, 3 uplink (UL) subframes, and one special subframe (SS).

On the DL, we use the cell specific LTE reference symbols (CS-RS) to estimate the channel. Linear interpolation in the frequency domain is used to obtain a channel estimate for the whole bandwidth. On the UL, the whole bandwidth is allocated to one single UE in subframe 4 and the corresponding demodulation reference symbols (DM-RS) are used to estimate the channel. Since these RS are available over the whole bandwidth, we do not need interpolation. More details on the measurement methodology can be found in [10].

b) Measurement Collection: One eNB with two antennas and one UE with one antenna was set up in Eurecom's lab (static setup). The eNB was collecting channel measurements for the UL while the UE was collecting channel measurements from the DL. The measured data was processed offline in Matlab. To estimate the reciprocity parameters, we used 10 consecutive samples of from the DL channel estimates from subframe 4 and the UL channel estimate from subframe 5.

c) Measurement Post-processing: Apart from the frequency offset, timing drifts are also present due offset between the clocks of the UE and eNB. The UE therefore needs to continuously adjust the frame start in order to stay synchronized with the eNB. These drifts and the resulting adjustments do not harm the normal (frame-based) modem operations. However, for the reciprocity calibration procedure they need to be reverted. Since these timing drifts are logged by the UE, they can be simply compensated by resampling the impulse responses (using a standard Lanczos filter) so that the peaks of the impulse responses are always on the same sample. 


\section{Performance Metrics}

The metric adopted here to evaluate the quality of the reciprocity-based CSIT estimation is again the achieved mutual information. Since MISO channels are considered, CSIT is exploited by doing maximum-ratio combining at the transmitter independently for each frequency of the 512 subcarriers of the OFDM modulation. The transmitter power is assumed constant over the considered bandwidth. The following mutual information expressions should be understood to be applied on a subcarrier basis. Denote the MISO channel for one subcarrier as $\mathbf{h}_{F}$ and its estimate with $\hat{\mathbf{h}}_{F}$. Assume that we want to transmit from A to B. We can distinguish three cases, depending on whether perfect, partial, or no channel state information is available at the transmitter (CSIT). For each case we evaluate the achievable mutual information:

1) The channel is known only at B: The optimal transmission strategy is Alamouti coding which achieves rate $R_{1}=\log _{2}\left(1+\frac{E_{S}}{N_{A} N_{0}} \mathbf{h}_{F} \mathbf{h}_{F}^{H}\right)$, where $E_{S} / N_{0}$ is the SNR and $N_{A}=2$ is the number of transmit antennas.

2) The channel is known to $A$ and $B$ : through optimal beamforming, rate $R_{2}=\log _{2}\left(1+\frac{E_{S}}{N_{0}} \mathbf{h}_{F} \mathbf{h}_{F}^{H}\right)$ is achievable.

3) A has only knowledge of $\hat{\mathbf{h}}_{F}$ (estimated from the reciprocity matrices) and $\mathrm{B}$ knows $\mathbf{h}_{F}$. We assume that we use the same transmission scheme as in case 2, but now the beamforming vector does not match the actual channel. Denote with $\mathbf{v}$ the right singular vector of $\hat{\mathbf{h}}_{F}$ corresponding to the largest singular value (optimal beamforming vector). Then $R_{3}=\log _{2}\left(1+\frac{E_{S}}{N_{0}} \mathbf{h}_{F} \mathbf{v} \mathbf{v}^{H} \mathbf{h}_{F}^{H}\right)$ is achievable.

Fig. 6 depicts the above performance metrics obtained by applying the estimated calibration factors to a part of the measurements taken 3 seconds after the calibration period. Additionally we also show the performance for the case where we do not perform any calibration, i.e., we assume that $\tilde{G}\left(t^{\prime}, \tau\right)=H\left(t^{\prime}, \tau\right)$; and for the case where we the calibration algorithm from [4] - oblivious to the frequency offset - is used. Remarkably, the capacity curve obtained with the channel reconstructed from the estimated calibration factors is almost as good as the one obtained for perfect CSIT. Furthermore, we see that neither of the two other methods perform well - their performance is almost the same as the case where there is no CSIT at all.

\section{CONCLUSION}

We have introduced a time-domain relative calibration algorithm robust to clock frequency offset between the nodes. This algorithm has been validated and benchmarked using synthetic signals. Furthermore, the proposed model has been validated though the use of bi-directional channel measurements. The proposed method and has been shown to provide accurate reciprocity-based CSIT in the presence of realistic impairments due to offsets between clock frequencies.

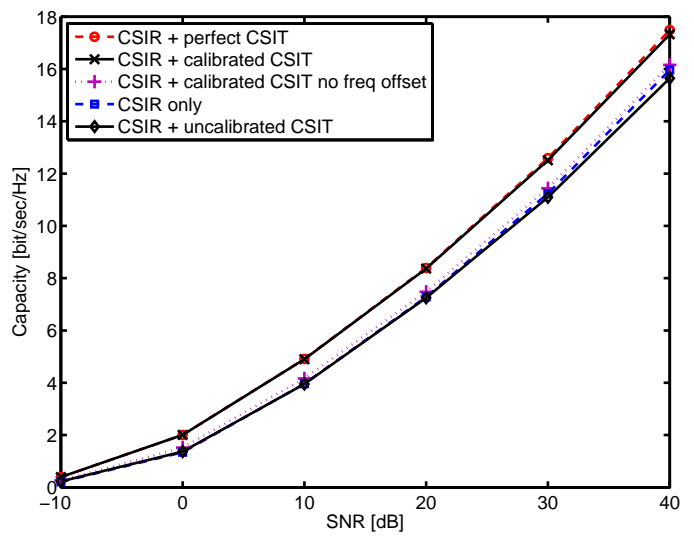

Fig. 6. Mutual information of the three cases: $G$ is known only at B (CSIR only), $G$ is known to A and B (CSIR + perfect CSIT), and A has only knowledge of $G_{\text {est }}$ (estimated from the reciprocity matrices) and B knows $G$ $(\mathrm{CSIR}+$ estimated CSIT).

\section{ACKNOWLEDGMENTS}

We acknowledge the support of the Franco-Austrian Hubert Curien Partnership "Amadeus", under grant \#FR05/2012. M. Guillaud was also supported by the FP7 HIATUS project of the European Commission (EC) and by the Austrian Science Fund (FWF) through grant NFN SISE (S106). F. Kaltenberger was also supported by the EC FP7 project SAMURAI.

\section{REFERENCES}

[1] Glenn S. Smith, "A direct derivation of a single-antenna reciprocity relation for the time domain," IEEE Transactions on Antennas and Propagation, vol. 52, no. 6, pp. 1568-1577, June 2004.

[2] A. Bourdoux, B. Côme, and N. Khaled, "Non-reciprocal transceivers in OFDM/SDMA systems: Impact and mitigation," in Proc. IEEE Radio and Wireless Conference (RAWCON), Boston, MA, USA, August 2003.

[3] V. Jungnickel, V. Krüger, G. Istoc, T. Haustein, and C. von Helmolt, "A MIMO system with reciprocal transceivers for the time-division duplex mode," in Proc. IEEE Antennas and Propagation Society Symposium, June 2004, vol. 2, pp. 1267-1270.

[4] M. Guillaud, D. T. M. Slock, and R. Knopp, "A practical method for wireless channel reciprocity exploitation through relative calibration," in Proc. Eighth International Symposium on Signal Processing and Its Applications (ISSPA '05), Sydney, Australia, August 2005.

[5] F. Kaltenberger, Haiyong Jiang, M. Guillaud, and R. Knopp, "Relative channel reciprocity calibration in MIMO/TDD systems," in Proc. Future Network \& Mobile Summit, Florence, Italy, June 2010.

[6] Shyamnath Gollakota, Samuel David Perli, and Dina Katabi, "Interference alignment and cancellation," in Proc. ACM SIGCOMM, New York, NY, USA, 2009, pp. 159-170, ACM.

[7] Per Zetterberg, "Experimental investigation of TDD reciprocity-based zero-forcing transmit precoding," EURASIP Journal on Advances in Signal Processing, vol. 2011, no. 137541, 2011.

[8] Nicola Mastronardi, Philippe Lemmerling, and Sabine Van Huffel, "Fast structured total least squares algorithm for solving the basic deconvolution problem," SIAM Journal on Matrix Analysis and Applications, vol. 22, pp. 533-553, 2000.

[9] C. Shepard, Hang Yu, N. Anand, E. Li, T. Marzetta, R. Yang, and Lin Zhong, "Argos: practical many-antenna base stations," in Proc. Mobile computing and networking (Mobicom) conference, Istanbul, Turkey, Aug. 2012, pp. 53-64.

[10] B. Zayen, B. Kouassi, R. Knopp, F. Kaltenberger, D. Slock, I. Ghauri, and L. Deneire, "Software implementation of spatial interweave cognitive radio communication using OpenAirInterface platform," in Proc. Int. Symposium on Wireless Communication Systems (ISWCS), Paris, France, Aug. 2012. 